\title{
BMJ Open Portable light therapy in the treatment of unipolar non-seasonal major depressive disorder: study protocol for the LUMIDEP randomised controlled trial
}

\author{
Eve Cosker (1) , ${ }^{1,2}$ Marie Moulard, ${ }^{1}$ Samuel Schmitt, ${ }^{1}$ Karine Angioi-Duprez, ${ }^{3}$ \\ Cédric Baumann, ${ }^{4}$ Vincent Laprévote, ${ }^{1,2}$ Raymund Schwan, ${ }^{1,2}$ Thomas Schwitzer ${ }^{1,2}$
}

To cite: Cosker E, Moulard M, Schmitt S, et al. Portable light therapy in the treatment of unipolar non-seasonal major depressive disorder: study protocol for the LUMIDEP randomised controlled trial. BMJ Open 2021;11:e049331. doi:10.1136/ bmjopen-2021-049331

- Prepublication history for this paper is available online. To view these files, please visit the journal online (http://dx.doi. org/10.1136/bmjopen-2021049331).

Received 21 January 2021 Accepted 25 June 2021

\section{Check for updates}

(C) Author(s) (or their employer(s)) 2021. Re-use permitted under CC BY-NC. No commercial re-use. See rights and permissions. Published by BMJ.

${ }^{1}$ Pôle Hospitalo-Universitaire De Psychiatrie d'Adultes et d'Addictologie Du Grand Nancy, Centre Psychothérapique de Nancy, Laxou, Lorraine, France ${ }^{2} \mathrm{U} 1114$, INSERM, 67085 Strasbourg, Alsace, France ${ }^{3}$ Département d'Ophtalmologie, Centre Hospitalier Universitaire de Nancy, Nancy, France

${ }^{4}$ Unité ESPRI-BioBase, Platforme PARC, CHRU de Nancy, Nancy, Lorraine, France

Correspondence to

Dr Eve Cosker;

eve.cosker@cpn-laxou.com

\section{ABSTRACT}

Introduction Major depressive disorder (MDD) affects more than 264 million people worldwide and is associated with an impaired quality of life as well as a higher risk of mortality. Current routine treatments demonstrate limited effectiveness. Light therapy (LT) on its own or in combination with antidepressant treatments could be an effective treatment, but the use of conventional LT devices use is restrictive. Portable LT devices allow patients to continue with their day-to-day activities and therefore encourage better treatment compliance. They have not been evaluated in MDD.

Methods and analysis The study is a single-centre, double-blind, randomised controlled trial assessing the efficacy of LT delivered via a portable device in addition to usual care (medical care and drug treatment) for inpatients and outpatients with unipolar non-seasonal MDD. Over the course of 8 weeks, patients use the device daily for 30 min at medium intensity as soon as possible after waking up and preferably between 07:00 and 09:00. All patients continue their usual care with their referring physician. $\mathrm{N}=50$ patients with MDD are included. The primary outcome measure is depressive symptom severity assessed using the Montgomery-Åsberg Depression Rating Scale between baseline and the eighth week. Secondary outcome measures are sleep quality assessed using the Pittsburgh Sleep Quality Index and Epworth Sleepiness Scale and anxiety level assessed on the Hamilton Anxiety Rating Scale, between baseline and week 8 . Further parameters relating to cognitive function are measured at baseline and after the intervention. An ancillary study aims to evaluate the impact of MDD on the retina and to follow its progression. Main limitations include risk of discontinuation or non-adherence and bias in patient selection.

Ethics and dissemination The study protocol was approved by lle de France X's Ethics Committee (protocol number 34-2018). Findings will be published in peerreviewed journals.

Trial registration number NCT03685942.

\section{INTRODUCTION}

Major depressive disorder (MDD) affects more than 264 million people worldwide and is the
Strengths and limitations of this study

- LUMIDEP is a prospective, randomised control trial.

- A major strength of the study is the use of a convincing placebo light therapy device.

- Another asset of the study is the regular evaluation of the symptoms of major depressive disorder and the follow-up after the therapeutic phase.

- Closeness to real-life situations is also a strength.

- Main limitations include possible loss to follow-up non-adherence and selection bias.

third most common cause of disability globally in terms of years lived with disability. ${ }^{1}$ It is associated with a deterioration in quality of life ${ }^{2}$ as well as a higher mortality risk. ${ }^{3}$ Currently, diagnosis of MDD is clinical and there are no relevant routine biomarkers available to doctors to assess diagnosis or guide treatment management. ${ }^{4}$ Current routine treatments-antidepressant drugs and psychotherapies-show limited effectiveness, with only $30 \%$ of patients responding to first antidepressant drug treatment. After trying multiple drug therapies, two-thirds of patients respond to treatment. ${ }^{5}$ Moreover, patients may experience side effects due to antidepressant treatment such as weight gain, sexual dysfunction, profuse sweating or a dry mouth, which persist in long-term use. ${ }^{6}$ Other therapeutic strategies and complementary examinations that can be used on a routine basis are therefore needed.

\section{Effectiveness of light therapy}

Light therapy (LT) is an effective treatment for seasonal affective disorder. ${ }^{7}$ On its own or in combination with antidepressant treatments, it could be an effective treatment for non-seasonal MDD. ${ }^{8}$ In fact, disruptions to the circadian rhythm such as irregular sleep/wake rhythms 
are observed in MDD and some hypotheses predict that endogenous circadian rhythm disturbances lead to MDD. ${ }^{9}$ Light is the most powerful time cue for circadian rhythmicity in humans and leads to rhythm synchronisation. The retina is the only light-sensory organ in the human body and transmits light information to the suprachiasmatic nucleus via the retinohypothalamic tract. ${ }^{10}$

Previously, McEnany and Lee found an improvement in MDD symptomatology among unmedicated female outpatients with MDD who used LT over a period of 26 days. ${ }^{11}$ However, the nature of the placebo, 'circadian adaptation glasses' that filter out light may be debated. Lam et al assessed the effect of LT and/or antidepressant drug among outpatients with unipolar non-seasonal MDD. ${ }^{12}$ The placebo device consisted of a deactivated negative ion generator. After 8 weeks, LT alone and in combination with drug treatment led to a significant change in MDD intensity. In the same way, in a monocentric, randomised double-blind study versus placebo, the proportion of patients attaining response and remission after 5 weeks was higher in the LT group. ${ }^{13}{ }^{14}$ However, the active or placebo groups used the device for different lengths of time, which may not guarantee double blinding. These results are consistent with the findings of Özdemir et al which also reported an improvement in anxiety symptoms. The study did not include a placebo LT device, however. ${ }^{15}$ Prasko et al obtained conflicting results, finding that after a short treatment period of 3 weeks, 2 hours of 5000 lux LT on its own was more effective than the combination of LT and imipramine, a tricyclic antidepressant. ${ }^{16}$ So, LT seems efficient alone or in combination with antidepressant. Moreover, LT might potentiate the action of antidepressant drugs, accelerate treatment response and reduce the time to remission. ${ }^{15}$ Only one study assessed if the effect of LT sustained after discontinuation. ${ }^{17}$ In fact, 5 weeks after discontinuation there was no more effect of LT.

To date, most studies have assessed conventional LT devices. They are stationary and require the patient to be immobile. However, portable LT devices including a holographic visor, which concentrates the light towards the pupil, have been available for several years now. They have not yet been evaluated in MDD, but preliminary results show some effectiveness in restoring sleep phases ${ }^{18}$ and improving cognitive performance. ${ }^{19}$ Unlike conventional devices, they allow to carry on with daily activities without impeding the view of the surroundings, thereby promoting better therapeutic compliance. We chose a naturalistic study design for drug treatment and do not arrange drug therapy. During the study, participants continue their usual care with their lead physician who decides on and prescribes the drug therapy. This allows the study to be as close as possible to routine practice.

The biggest challenge for studies assessing the efficacy of LT lies in providing a convincing placebo device. In fact, if the light emitted by the placebo device is insufficient or a different colour to the active device, group blinding cannot be guaranteed, and patients may find out which group they have been assigned to. If the light from the placebo device is too intense, though, it could become active, meaning there would no longer be a placebo group. To be able to guarantee blinding, in our study, the active and placebo devices are visually identical and are used for the same duration.

\section{Retinal assessment in MDD}

In the field of psychiatric diseases, developing routine examinations is challenging. The exploration of brain function remains complex due to the brain's location in the cranial cavity. However, the retina, as an extension of the central nervous system due to embryonic development, ${ }^{20}$ appears to be important site for investigating neurotransmission anomalies in neuropsychiatric pathologies. ${ }^{21-23}$ Moreover, the retina is easy to access and can be assessed using non-invasive paraclinical examination methods such as electroretinogram (ERG) or optical coherence topography (OCT). For example, Bubl et alfound a decrease in pattern ERG (PERG) contrast gain in patients with MDD, independently of antidepressant treatment, compared with controls. ${ }^{24}$ Follow-up demonstrates that the patients who did not achieve remission retained a significantly lower PERG contrast gain while it normalised in the patients who responded, suggesting that PERG contrast gain could serve as an MDD state marker. ${ }^{25}$ In the same way, Fornaro et al found that patients with MDD who responded to duloxetine, a serotonin-norepinephrine reuptake inhibitor antidepressant, had specific changes in the function of the first layer of the retina, compared with non-responding patients. ${ }^{26}$ This would seem to imply the existence of retinal markers of MDD status and prediction of the therapeutic response. We chose to assess retinal function at different points in the protocol in order to manage its progression and to use the standardised International Society for Clinical Electrophysiology of Vision protocol to assess whole retina function and make our results replicable and comparable. ${ }^{27}$

\section{Objectives}

The first and main aim is to assess the effectiveness of LT provided by a portable device in combination with usual care (medical care and drug treatment) compared with usual care for unipolar, non-seasonal MDD. Second, we will assess the effectiveness of the LT provided by the portable device on sleep quality and anxiety level. The ancillary study aims to evaluate impact of MDD on the retina and to monitor its progression.

\section{METHODS AND ANALYSIS}

\section{Study design}

The study taking place in the Nancy Psychotherapeutic Center, France, is a monocentric double-blind, randomised controlled trial in which participants are either randomised into the LT group or into the placebo group, in a parallel group design. Randomisation is individual with a 1:1 allocation ratio using block randomisation. No stratification is planned. The randomisation list was generated by a computer under the supervision of the study's methodologist before the start of the study. After inclusion, based on the order in which he is enrolled in the study, the participant is assigned a randomisation number 
determining group allocation. The trial is registered at ClinicalTrials.gov. The study protocol was approved by the Ile de France X Ethics Committee (no. 34-2018). This is the fourth version of the protocol, 13 July 2020. The study is ongoing. The study started in January 2019 and is expected to complete by September 2023. A flow diagram of the study is provided in figure 1.

\section{Setting, recruitment and enrolment}

Patients are recruited from inpatients and outpatients of the Nancy Psychotherapeutic Center and from outpatients of physicians in private practice near the recruitment centre. Doctors are informed of the study and may refer their patients. Posters and flyers are distributed within the Nancy Psychotherapeutic Center and in private practices. Information is also available on social media via the Nancy Psychotherapeutic Center's profile.

During appointment for baseline assessment, the patient is provided with detailed oral and written information about main and ancillary studies by a trained psychiatrist. The patient is given the opportunity to ask questions and consider whether to take part. In the event that the patient wishes to participate, they give their written informed consent. Then, the patient is screened for inclusion and exclusion criteria. If the patient meets all the criteria, he is included in the study.

\section{Inclusion and exclusion criteria}

The inclusion criteria are:

1. MDD diagnosis according to the Diagnostic and Statistical Manual of Mental Disorders IV (DSMIV), assessed by means of the Mini Neuropsychiatric International Interview (MINI) ${ }^{28}$

2. Age between 18 and 60 years.

3. Affiliation with a welfare scheme.

4. Complete information on the study received and written informed consent signed

The exclusion criteria are:

1. Diagnosis of a progressive psychiatric disorder (except MDD and anxiety disorder) according to Axis I of the DSM-IV, assessed by means of the MINI. ${ }^{28}$

2. Absence of routine care for MDD.

3. Previous or current LT treatment.

4. Seasonal affective disorder.

5. High suicide risk, assessed by means of the MINI. ${ }^{28}$

6. Ongoing neurological disease.

7. Retinal pathology.

8. Participation in another study.

9. Patient impairment leading to difficulty participating or impossibility or inability to understand the information provided on the study.

10. Persons cited in Articles L. $1121-5$ to L. $1121-8$ of the French Public Health Code: pregnant women, parturient or breastfeeding mothers, persons deprived of their liberty by a judicial or administrative decision, persons under psychiatric care under duress, persons admitted to a health or social establishment for other goals than research, minors, adults subject to a legal protection,

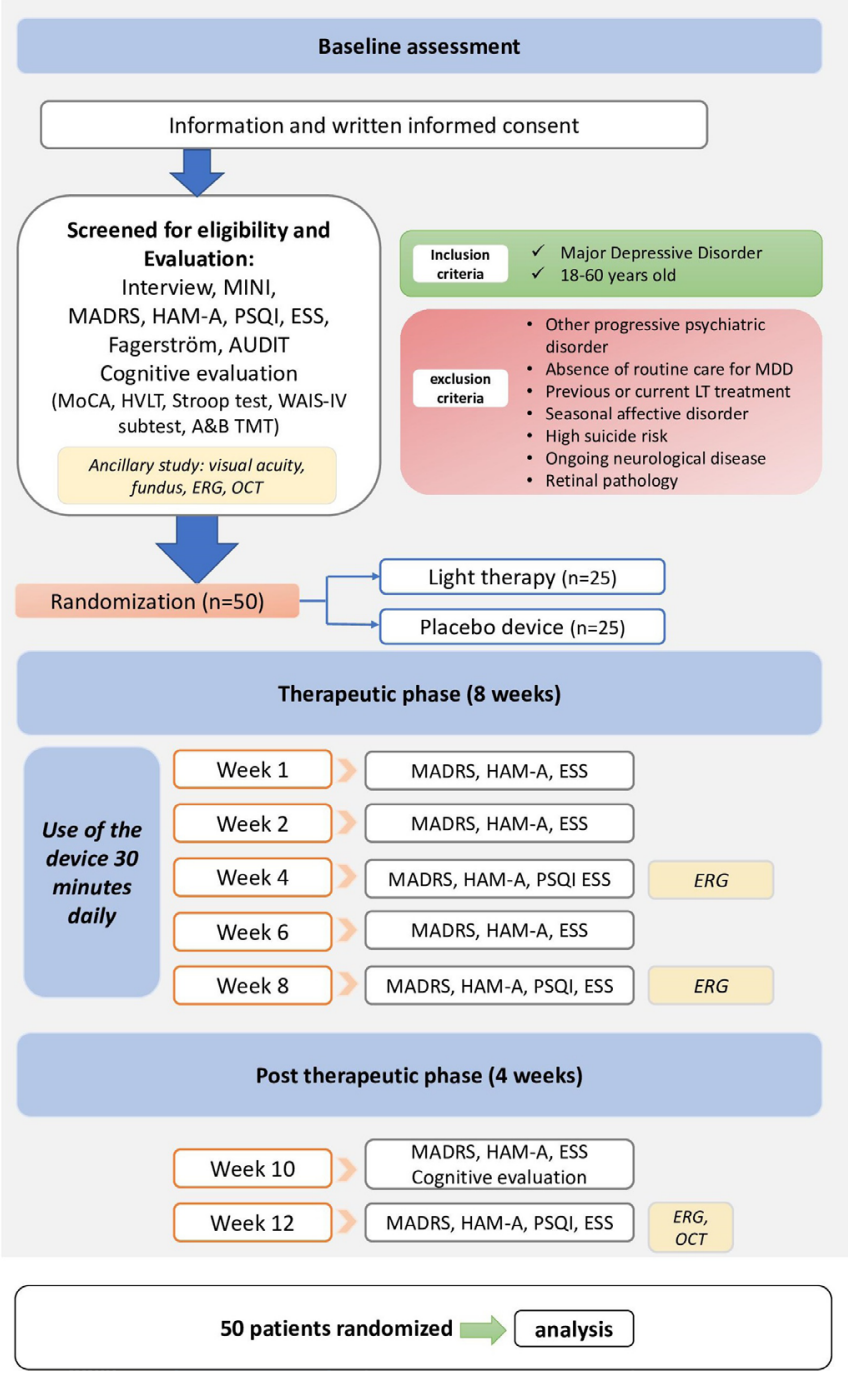

Figure 1 Flow diagram of the study. Participants included in the study are randomised to the LT group or to the placebo group. During the treatment phase participants use the device for 30 min daily at medium intensity, as soon as possible after waking up and preferably between 07:00 and 09:00. In the post-therapeutic phase, they will no longer use the device. During the study, participants continue their usual care. Fifty patients will be included in the trial. A\&B TMT, A and B Trail Making Test; AUDIT, Alcohol Use Disorder Identification Test; ESS, Epworth Sleepiness Scale; Fagerström, Fagerström test; HAM-A, Hamilton Anxiety Rating Scale; HVLT, Hopkins Verbal Learning Test; LT, light therapy; MADRS, Montgomery-Åsberg Depression Rating Scale; MDD, major depressive disorder; MINI, Mini Neuropsychiatric International Interview; MoCA, Montreal Cognitive Assessment; PSQI, Pittsburgh Sleep Quality Index; WAIS-IV subtest, Weschler Adult Intelligence Scale Digit Span subtest; the yellow rectangles are for the ancillary study: ERG, electroretinogram; OCT, optical coherence tomography.

adults who are unable to express their consent and who are not subject to a legal protection measure.

\section{Intervention}

Patients who are included are randomised either to the LT group and are given an active LT device or to the 
placebo group and receive a placebo LT device. The study is split into two phases. The first phase is the treatment phase and lasts for 8 weeks after inclusion. During this phase, patients will use the device for $30 \mathrm{~min}$ daily at medium intensity, as soon as possible after waking up and preferably between 07:00 and 09:00. The second phase is the post-therapeutic phase, during which patients will no longer use the device. The second phase starts immediately after the first phase and lasts 4 weeks. The total duration of the study per patient adds up to 12 weeks.

At the end of the inclusion visit, the patient receives an active or a placebo LT device, depending on their randomisation group. Use of the device starts the day after inclusion. Outpatient participants use the device at home and inpatient at the hospital. In order to monitor adherence, the patient is given a follow-up notebook in order to make a daily record of whether the device has been used, as well as the start and end times of use. The device is used in addition to usual care for patients with MDD.

\section{Device characteristics}

The active portable LT device is Luminette produced by Lucimed SA, Villers-Le-Bouillet, Belgium. It is a pair of holographic glasses worn like a visor that provide white light enriched with blue with a wavelength of $468 \mathrm{~nm}$. The present study uses medium intensity (intensity 2). Light intensity is 1000 lux.

The placebo portable device looks identical to the active device but emits white light with a wavelength of $660 \mathrm{~nm}$ without enriched blue light, which does not affect circadian rhythms. Light intensity is 175 lux. It is produced by Lucimed SA, Villers-Le-Bouillet, Belgium.

\section{Concomitant care}

Participants continue to receive their usual care from their lead physician throughout the duration of the study. Patients' drug treatment is documented in terms of type and dosage at each visit over the entire course of the study. In order to avoid biases, all study participants are asked not to use any other LT device while they are participating in the study.

\section{Measures}

Primary and secondary outcome measures

We chose the Montgomery-Åsberg Depression Rating Scale (MADRS) ${ }^{29}$ as the primary outcome measure to capture the change in MDD symptom severity between baseline and the eighth week of the study. The total score varies between 0 and 27. A score of $\leq 12$ means an absence of a depressive episode, from 13 to 19 a mild depressive episode and $>20$ a moderate or major episode.

Secondary outcome measures are sleep quality and anxiety. Sleep quality is assessed at baseline and after 8 weeks based on two scores. The first one is the Pittsburgh Sleep Quality Index (PSQI) ${ }^{30}$ which is composed of 11 rated items. A score of $>5$ expresses a sleep disturbance. The second score used is the Epworth Sleepiness Scale (ESS) ${ }^{31}$ This score is self-rated and composed of eight items, with the total score rated out of 24 . A score of $\geq 15$ means excessive daytime sleepiness.

Anxiety is assessed based on the 14 items of the Hamilton Anxiety Rating Scale (HAM-A) ${ }^{32}$ A score of $<17$ indicates mild anxiety, a score of between 18 and 24 indicates medium-to-moderate anxiety and a score of between 25 and 30indicates moderate-to-severe anxiety.

\section{Ancillary study outcome measures}

Retinal function will be assessed via an ERG performed at baseline and after 4, 8 and 12 weeks, and an OCT done at baseline (within \pm 72 hours) and after 12 weeks (within \pm 72 hours).

\section{Further measures}

Cognitive function may be impacted in MDD and this impairment may persist after remission and be disabling for patients. ${ }^{33}$ Cognitive function is therefore assessed at baseline and at week 10 using the following scales: the Montreal Cognitive Assessment, which evaluates neurocognitive impairment, ${ }^{34}$ the Hopkins Verbal Learning Test, which evaluates verbal learning and memory, ${ }^{35}$ the Stroop Test, which evaluates selective attention, ${ }^{36}{ }^{37}$ the A and B Trail Making Test, which evaluates executive functions $^{38}$ and the Weschler Adult Intelligence Scale (WAIS-IV) Digit Span subtest, which evaluates working memory. ${ }^{39}$

\section{Baseline assessment}

An interview and a clinical examination are conducted, including socio-demographic data: age, gender, height, weight, level of academic achievement, tobacco consumption in cigarettes per day and alcohol consumption. MINI is used to diagnose MDD. ${ }^{28}$ The severity of the MDD is self-rated using MADRS ${ }^{29}$ and the anxiety level is interview-rated based on the HAM-A. ${ }^{32}$ Sleep quality is self-assessed at baseline using the PSQI ${ }^{30}$ and the ESS. ${ }^{31}$ For patients who smoke, the level of tobacco addiction is assessed using the Fagerström test. ${ }^{40}$ Alcohol abuse is evaluated by means of the Alcohol Use Disorder Identification Test. ${ }^{41}$ Cognitive function is assessed as described above. All scales are completed using a paper and pencil.

The participation in the ancillary study is proposed to all patients of the main study. Its additional inclusion criteria are normal corrected visual acuity measured using the Monoyer chart and normal fundus examination. They will be given an ERG and, within 72 hours, an OCT. This medical examination will be performed by an experienced ophthalmologist in the ophthalmology department of the university-affiliated regional centre in Nancy.

\section{Patient follow-up and data collection Treatment phase}

Five visits are planned at 1 week (within \pm 1 day) (W1), 2 weeks (W2) ( \pm 1 day), 4 weeks (W4) ( \pm 2 days), 6 weeks (W6) ( \pm 2 days) and 8 weeks (W8) (within 4 days afterwards) after baseline evaluation. Each visit involves a physical examination and a clinical interview, performed by a 
Table 1 Overview on time point of measurement and outcomes

\begin{tabular}{|c|c|c|c|c|c|c|c|c|c|}
\hline & & \multirow[b]{2}{*}{ Do } & \multicolumn{5}{|c|}{ Treatment phase } & \multicolumn{2}{|c|}{ Post-therapeutic phase } \\
\hline & & & $\begin{array}{l}\text { W1 ( } \pm 1 \\
\text { day) }\end{array}$ & $\begin{array}{l}\text { W2 ( } \pm 1 \\
\text { day) }\end{array}$ & $\begin{array}{l}\text { W4 ( } \pm 2 \\
\text { days) }\end{array}$ & $\begin{array}{l}\text { W6 ( } \pm 2 \\
\text { days) }\end{array}$ & $\begin{array}{l}\text { W8 (+4 } \\
\text { days) }\end{array}$ & W10 ( \pm 2 days) & W12 ( \pm 2 days) \\
\hline \multicolumn{10}{|c|}{ Primary outcome } \\
\hline Depression & MADRS & $x$ & $\mathrm{X}$ & $\mathrm{X}$ & $\mathrm{X}$ & $x$ & $\mathrm{X}$ & $x$ & $x$ \\
\hline \multicolumn{10}{|c|}{ Secondary outcomes } \\
\hline Sleep quality & PSQI & $x$ & & & $x$ & & $x$ & & $x$ \\
\hline Anxiety & HAM-A & $x$ & $x$ & $X$ & $X$ & $x$ & $x$ & $x$ & $\mathrm{X}$ \\
\hline
\end{tabular}

Further measures

\begin{tabular}{|c|c|c|c|c|c|c|c|c|c|}
\hline \multirow[t]{4}{*}{ Cognitive functions } & MoCA & $x$ & & & & & & \multicolumn{2}{|l|}{$x$} \\
\hline & HVLT & $x$ & & & & & & \multicolumn{2}{|l|}{$\mathrm{x}$} \\
\hline & Stroop test & $x$ & & & & & & \multicolumn{2}{|l|}{$x$} \\
\hline & A\&B TMT & $x$ & & & & & & \multicolumn{2}{|l|}{$\mathrm{x}$} \\
\hline \multicolumn{2}{|l|}{ Physical examination } & $x$ & $x$ & $x$ & $x$ & $x$ & $x$ & $x$ & $x$ \\
\hline
\end{tabular}

Ancillary's study outcomes

\begin{tabular}{|c|c|c|c|c|c|}
\hline \multirow[t]{3}{*}{ Retinal function } & $\mathrm{FE}$ & $x$ & \multirow[b]{3}{*}{$x$} & \multirow[b]{3}{*}{$x$} & \multirow[b]{3}{*}{$x$} \\
\hline & Visual acuity & $x$ & & & \\
\hline & ERG & $x$ & & & \\
\hline \multicolumn{6}{|c|}{ Baseline assessment } \\
\hline \multicolumn{2}{|c|}{$\begin{array}{l}\text { MINI } \\
\text { AUDIT } \\
\text { Fagerström test }\end{array}$} & $x$ & & & \\
\hline
\end{tabular}

Note: $\mathrm{D} 0=$ baseline measure; $\mathrm{W} 1=$ evaluation 1 week after baseline measure within \pm 1 day; and so on.

A\&B TMT, A and B Trail Making Test; AUDIT, Alcohol Use Disorder Identification Test; ERG, electroretinogram; ESS, Epworth Sleepiness Scale; FE, fundus examination; HAM-A, Hamilton Anxiety Rating Scale; HVLT, Hopkins Verbal Learning Test; MADRS, Montgomery-Åsberg Depression Rating Scale; MINI, Mini Neuropsychiatric International Interview; MoCA, Montreal Cognitive Assessment; OCT, optical coherence tomography; PSQI, Pittsburgh Sleep Quality Index; WAIS-IV subtest, Weschler Adult Intelligence Scale Digit Span subtest.

trained psychiatrist. MDD intensity is evaluated at each visit using the MADRS, anxiety level using the HAM-A and sleep quality using the ESS. Sleep quality is further evaluated at W4 and W8 visits using the PSQI. For patients participating in the ancillary study, retinal function is evaluated by means of an ERG at W4 and W8.

\section{Post-therapeutic phase}

Two visits are planned, 10 weeks (W10) $( \pm 2$ days) and 12 weeks (W12) ( \pm 2 days) after baseline evaluation. A physical examination and a clinical interview are conducted by a trained psychiatrist, to evaluate MDD intensity using the MADRS and anxiety level using the HAM-A. Sleep quality is rated using the ESS and is further evaluated at W12 using the PSQI. Cognitive function is assessed at W10 as described above. For patients participating in the ancillary study, retinal function is evaluated using an ERG at W12 and an OCT at W12 (within \pm 72 hours).

All measurements taken during the study are summarised in table 1 .

\section{Safety evaluation}

At each visit the interview explores possible adverse effects and their presence or absence will be recorded in the study case report form. If adverse events are related to health vigilance, the investigator will forward them to the relevant health vigilance institution according to the usual French reporting procedure. Any patient experiencing an adverse reaction receives the appropriate treatment and is monitored until the effect is resolved or until the end of the research. If necessary, use of the device may be discontinued. The known side effects of LT are transient and of low intensity: headache, nausea and eye fatigue.

\section{Blinding}

The double-blind is guaranteed because neither the patient nor the investigator is able to find out which group the patient belongs to. The placebo and the active device are identical. A difference in brightness intensity might be perceived during activation but the patient is 
not aware of it. To preserve the blind for the investigator, the device will be stored before baseline, managed and dispensed by the Nancy Psychotherapeutic Center pharmacist. This way, the investigators will never handle the device. Investigators do not have access to the centralised randomisation list during the study. During the course of the research, the blind may be lifted if and only if the participant's medical condition requires it.

\section{Rules for discontinuation and drop-out criteria}

Drop-outs from the study are permanent. They occur when the patient wishes to discontinue their participation in the study. The investigator may temporarily or permanently discontinue an individual's participation in the research for any reason that affects the patient's safety or would be in the patient's best interests or in case of lack of use of the device. The patient is considered lost to follow-up when the investigator has no news of them. In case of drop-out, the patient is replaced by another one.

\section{Sample size}

Based on the data in the literature, we expect an average score for depressive symptoms in the placebo group of at least 18 points at $\mathrm{W} 8(\mathrm{SD}=9) .{ }^{12}$ To demonstrate a clinically relevant decrease in the minimum average score of 8 points in the LT group, meaning an expected average score of $10(\mathrm{SD}=9)$ at $\mathrm{W} 8$, in a unilateral situation with alpha risk set at $5 \%$ and a statistical power of $90 \%$, it is necessary to analyse 22 patients per group (1:1 ratio). We plan to include $10 \%$ more patients to offset any possible lack of data. Lastly, 50 patients will be included in the trial (25 per group).

\section{Statistical analysis}

The parameters collected are described by the usual indicators: percentage for categorical variables, mean, SD, quartiles, outliers and distribution for continuous variables. Comparisons of the main and secondary outcomes are performed using Student's t-test on paired series (or the signed rank test) and the McNemar test. In the case of significant differences between groups immediately after randomisation, an adjustment is made using conditional regression models. Intention-to-treat and then per-protocol analyses are performed. No interim analysis is planned. The threshold of statistical significance is set at $5 \%$. No imputation of missing values is planned. If the number of missing values becomes relevant (main outcome, number of missing data, imbalance, etc), a possible method for imputing medical data may be planned and described in the statistical analysis plan.

\section{Ethics and dissemination}

The trial is registered at ClinicalTrials.gov. This trial is registered with the French National Agency for the Safety of Medicines and Health Products (ID-RCB: 2017-A0334944). The study protocol was approved by Ile de France X's Ethics Committee (protocol number 34-2018). Changes to the protocol are reported to the committee. The procedure will be performed following the principles described in the declaration of Helsinki. We will publish the results of this study in peer-reviewed journals.

\section{No patient and public involvement}

There were no founds or time allocated for patient and public involvement.

\section{DISCUSSION}

This randomised controlled trial aims to assess the effectiveness of LT provided by a portable device for a period of 8 weeks, in combination with usual care (medical care and drug treatment) compared with usual care for unipolar non-seasonal MDD.

Considering the high prevalence of MDD worldwide and the difficulties encountered with existing treatments, ${ }^{5}{ }^{42}$ an efficacious LT portable device for MDD would provide numerous benefits by strengthening the therapeutic arsenal and by potentiating the effectiveness of antidepressants already used in current practice. In previous studies, the duration of LT varies from 3 to 8 weeks. ${ }^{12}{ }^{14-16}$ However, after the discontinuation of LT after 5 weeks of treatment there seems to be no more effect of LT. ${ }^{17}$ So, based on this and the results of Lam $e t$ $a l^{12}$ and Özdemir $e t a l,{ }^{15}$ we choose to assess the efficacy of LT after 8 weeks.

The known side effects of LT are transient, of low intensity and uncommon: headache, nausea and eye strain. ${ }^{43}$ LT therefore has a very favourable benefit/risk ratio. LT is of particular interest in populations for whom the prescription of antidepressants may pose a risk or is contraindicated, such as for children, pregnant or breastfeeding women or the elderly. A few studies have already suggested LT as a possible treatment for children and adolescents, ${ }^{44}{ }^{45}$ patients aged 60 or older ${ }^{46} 47$ and pregnant women. ${ }^{48}$

We chose to assess the efficacy of a portable LT device providing 1000 lux white light enriched with blue with a wavelength of $468 \mathrm{~nm}$. In fact, in seasonal affective disorder several studies demonstrated that blue enriched light at low intensity (750 lux) are as effective as standard full spectrum light therapy at 10000 lux intensity. ${ }^{49-51}$ This might be underlined by the greater sensitivity of the human circadian system to short wavelengths than to long wavelengths. ${ }^{49}$ Indeed, short wavelengths are a stimulator of melanopsin-containing intrinsically photosensitive retinal ganglion cells. ${ }^{51}$

Moreover, the portable device may prevent some of the drawbacks of conventional LT such as the impossibility of movement and the difficulty of continuing with day-to-day activities during treatment. The length of the session is another possible limitation for the use of light therapy, but the portable LT device may favour the use of LT given how easy it is to use, which makes it possible to carry on with day-to-day activities during the LT session. We therefore believe that portable devices enable better therapeutic compliance. 
The study complies with methodological standards such as randomisation and a placebo group and provides a convincing placebo device. Additionally, we added a post-therapeutic follow-up to assess progression posttreatment. Another strength of this study is its closeness to real-life situations. Indeed, LT is used in combination with usual care. Since the treatments approximate actual practice, the results will have strong external validity. Furthermore, the socio-demographic assessment is intended to be as complete as possible in order to control confounding factors. This study has several limitations. First, it is a single centre study. Second, this study is time-consuming for patients. As loss of motivation is a symptom of MDD, it may lead to discontinuation of the study or to a bias in patient selection. In order to facilitate patient participation, visits are planned in, allowing for minor leeway, in line with the patient's schedule. Third, loss of motivation may also lead to the non-use of the device. To monitor adherence, patients are asked to record daily in a notebook whether or not they used the device and duration of use. However, the monitoring of adherence remains declaratory. Fourth, the follow-up post-therapeutic phase lasts 1 month which means that in case of effectiveness we cannot evaluate its persistence beyond this month. Finally, the study does not control or measure the patient's naturalistic light exposure.

The ancillary study aims to evaluate the impact of MDD on retinal structure and function and to follow its progression during the treatment phase. To date, MDD diagnosis has been clinical and patients do not benefit from routine complementary examinations that could confirm the diagnosis, guide the choice of treatment and manage treatment efficacy. The retina is of interest and it has already been suggested that MDD may impact retinal function. ${ }^{823}$

Previous studies using ERG found that assessing the first retinal layers may provide clues to predict treatment response and effectiveness. ${ }^{26}$ Moreover, the last retinal layer assessment may be a state marker of MDD. ${ }^{25}$ It is pertinent to study them jointly. Regarding structural impairment of the retina during MDD, studies highlight that duration and recurrence may impair retinal structure ${ }^{52}{ }^{53}$ but this needs further verification. ${ }^{54}{ }^{55}$ To date, though, no study has ever combined the assessment of all layers and the structure of the retina and managed its progression throughout the course of the treatment.

In conclusion, considering the randomisation and the use of a convincing placebo LT device that is visually identical to the active LT device, the LUMIDEP study will determine if portable LT devices can be added to the therapeutic arsenal for the treatment of unipolar nonseasonal MDD.

Contributors TS and EC were responsible for and the main contributors to the concept, design and conduct of the study and made a substantial contribution to the development of the research question and the writing of the manuscript. MM and SS contributed substantially to the writing of the manuscript and conduct of the study. KA-D contributed to the ancillary part of the study and to the writing of manuscript for that part. RS and VL contributed to the development of the research question and to the writing of the manuscript. $C B$ contributed to the design of the data analysis for the study and to the writing of the data analysis section of the manuscript. All authors read and approved the final manuscript. No professional writers were hired.

Funding This study is funded jointly by the Nancy Psychotherapeutic Center, 1 rue du Docteur Archambault, 54520 Laxou, France, +33383925349, unic@cpn-laxou. com and LUCIMED SA, Villers-Le-Bouillet, Belgium.

Competing interests None declared.

Patient and public involvement Patients and/or the public were not involved in the design, or conduct, or reporting, or dissemination plans of this research.

Patient consent for publication Not required.

Provenance and peer review Not commissioned; externally peer reviewed.

Open access This is an open access article distributed in accordance with the Creative Commons Attribution Non Commercial (CC BY-NC 4.0) license, which permits others to distribute, remix, adapt, build upon this work non-commercially, and license their derivative works on different terms, provided the original work is properly cited, appropriate credit is given, any changes made indicated, and the use is non-commercial. See: http://creativecommons.org/licenses/by-nc/4.0/.

ORCID iD

Eve Cosker http://orcid.org/0000-0002-0060-7529

\section{REFERENCES}

1 GBD 2017 Disease and Injury Incidence and Prevalence Collaborators. Global, regional, and national incidence, prevalence, and years lived with disability for 354 diseases and injuries for 195 countries and territories, 1990-2017: a systematic analysis for the global burden of disease study 2017. Lancet 2018;392:1789-858.

2 Ishak WW, Ha K, Kapitanski N, et al. The impact of psychotherapy, pharmacotherapy, and their combination on quality of life in depression. Harv Rev Psychiatry 2011;19:277-89.

3 Gilman SE, Sucha E, Kingsbury M, et al. Depression and mortality in a longitudinal study: 1952-2011. Can Med Assoc J 2017;189:E1304-10.

4 Niciu MJ, Mathews DC, Nugent AC, et al. Developing biomarkers in mood disorders research through the use of rapid-acting antidepressants. Depress Anxiety 2014;31:297-307.

5 Duman RS, Stress Nof. Neurobiology of stress, depression, and rapid acting antidepressants: remodeling synaptic connections. Depress Anxiety 2014;31:291-6.

6 Bet PM, Hugtenburg JG, Penninx BWJH, et al. Side effects of antidepressants during long-term use in a naturalistic setting. Eur Neuropsychopharmacol 2013;23:1443-51.

7 Westrin A, Lam RW. Long-Term and preventative treatment for seasonal affective disorder. CNS Drugs 2007;21:901-9.

8 Cosker E, Schwan R, Angioi-Duprez K, et al. New insights on the role of the retina in diagnostic and therapeutic strategies in major depressive disorder. Neurosci Biobehav Rev 2020;113:262-72.

9 Vadnie CA, McClung CA. Circadian rhythm disturbances in mood disorders: insights into the role of the suprachiasmatic nucleus. Neural Plast 2017;2017:1-28.

10 Pévet P. The internal time-giver role of melatonin. A key for our health. Rev Neurol 2014;170:646-52.

11 McEnany GW, Lee KA. Effects of light therapy on sleep, mood, and temperature in women with nonseasonal major depression. Issues Ment Health Nurs 2005;26:781-94.

12 Lam RW, Levitt AJ, Levitan RD, et al. Efficacy of bright light treatment, fluoxetine, and the combination in patients with Nonseasonal major depressive disorder: a randomized clinical trial. JAMA Psychiatry 2016;73:56-63.

13 Martiny K. Adjunctive bright light in non-seasonal major depression. Acta Psychiatr Scand Suppl 2004:7-28.

14 Martiny K, Lunde M, Undén M, et al. Adjunctive bright light in nonseasonal major depression: results from clinician-rated depression scales. Acta Psychiatr Scand 2005;112:117-25.

15 Güzel Özdemir P, Boysan M, Smolensky MH, et al. Comparison of venlafaxine alone versus venlafaxine plus bright light therapy combination for severe major depressive disorder. J Clin Psychiatry 2015;76:e645-54.

16 Prasko J, Horacek J, Klaschka J, et al. Bright light therapy and/or imipramine for inpatients with recurrent non-seasonal depression. Neuro Endocrinol Lett 2002;23:109-13. 
17 Martiny K, Lunde M, Undén M, et al. The lack of sustained effect of bright light, after discontinuation, in non-seasonal major depression. Psychol Med 2006;36:1247-52.

18 Langevin $\mathrm{RH}$, Laurent $\mathrm{A}$, Sauvé Y. Évaluation préliminaire de

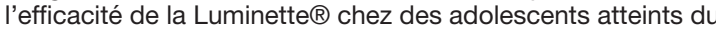
syndrome de retard de phase du sommeil (SRPS) : essai randomisé en simple insu et contrôlé par placebo. Médecine du Sommeil 2014;11:91-7.

19 Slama H, Deliens G, Schmitz R, et al. Afternoon nap and bright light exposure improve cognitive flexibility post lunch. PLoS One 2015;10:e0125359.

20 Hoon M, Okawa H, Della Santina L, et al. Functional architecture of the retina: development and disease. Prog Retin Eye Res 2014;42:44-84.

21 Lavoie J, Illiano P, Sotnikova TD, et al. The electroretinogram as biomarker of central dopamine and serotonin: potential relevance to psychiatric disorders. Biol Psychiatry 2014;75:479-86.

22 London A, Benhar I, Schwartz M. The retina as a window to the brain-from eye research to CNS disorders. Nat Rev Neurol 2013;9:44-53.

23 Schwitzer T, Lavoie J, Giersch A, et al. The emerging field of retinal electrophysiological measurements in psychiatric research: a review of the findings and the perspectives in major depressive disorder. $J$ Psychiatr Res 2015;70:113-20.

24 Bubl E, Kern E, Ebert D, et al. Seeing gray when feeling blue? depression can be measured in the eye of the diseased. Biol Psychiatry 2010;68:205-8.

25 Bubl E, Ebert D, Kern E, et al. Effect of antidepressive therapy on retinal contrast processing in depressive disorder. Br J Psychiatry 2012;201:151-8.

26 Fornaro M, Bandini F, Ogliastro C, et al. Electroretinographic assessment in major depressed patients receiving duloxetine: might differences between responders and non-responders indicate a differential biological background? J Affect Disord 2011;135:154-9

27 Robson AG, Nilsson J, Li S, et al. ISCEV guide to visual electrodiagnostic procedures. Doc Ophthalmol 2018;136:1-26.

28 Sheehan DV, Lecrubier Y, Sheehan KH, et al. The Mini-International neuropsychiatric interview (M.I.N.I.): the development and validation of a structured diagnostic psychiatric interview for DSM-IV and ICD10. J Clin Psychiatry 1998;59 Suppl 20:22-33.

29 Montgomery SA, Asberg M. A new depression scale designed to be sensitive to change. Br J Psychiatry 1979;134:382-9.

30 Buysse DJ, Reynolds CF, Monk TH, et al. The Pittsburgh sleep quality index: a new instrument for psychiatric practice and research Psychiatry Res 1989;28:193-213.

31 Johns MW. A new method for measuring daytime sleepiness: the Epworth Sleepiness scale. Sleep 1991;14:540-5

32 Hamilton M. The assessment of anxiety states by rating. Br J Med Psychol 1959;32:50-5.

33 Knight MJ, Baune BT. Cognitive dysfunction in major depressive disorder. Curr Opin Psychiatry 2018;31:26-31.

34 Nasreddine ZS, Phillips NA, Bédirian V, et al. The Montreal cognitive assessment, MoCA: a brief screening tool for mild cognitive impairment. J Am Geriatr Soc 2005;53:695-9.

35 Rieu D, Bachoud-Lévi A-C, Laurent A, et al. [French adaptation of the Hopkins Verbal Learning Test]. Rev Neurol 2006;162:721-8.

36 Scarpina F, Tagini S. The Stroop color and word test. Front Psychol 2017;8:557.
37 Stroop JR. Studies of interference in serial verbal reactions. J Exp Psychol 1935;18:643-62.

38 Tombaugh TN. Trail making test a and B: normative data stratified by age and education. Arch Clin Neuropsychol 2004;19:203-14.

39 Jasinski LJ, Berry DTR, Shandera AL, et al. Use of the Wechsler adult intelligence scale digit span subtest for malingering detection: a meta-analytic review. J Clin Exp Neuropsychol 2011;33:300-14.

40 Heatherton TF, Kozlowski LT, Frecker RC, et al. The Fagerström test for nicotine dependence: a revision of the Fagerström tolerance questionnaire. Br J Addict 1991;86:1119-27.

41 Saunders JB, Aasland OG, Babor TF, et al. Development of the Alcohol Use Disorders Identification Test (AUDIT): WHO Collaborative Project on Early Detection of Persons with Harmful Alcohol Consumption--Il. Addiction 1993;88:791-804.

42 Smith K. Mental health: a world of depression. Nature 2014;515:180-1.

43 Oldham MA, Ciraulo DA. Bright light therapy for depression: a review of its effects on chronobiology and the autonomic nervous system. Chronobiol Int 2014;31:305-19.

44 Giedd JN, Swedo SE, Lowe CH, et al. Case series: pediatric seasonal affective disorder. A follow-up report. J Am Acad Child Adolesc Psychiatry 1998;37:218-20.

45 Kirschbaum-Lesch I, Gest S, Legenbauer T, et al. Feasibility and efficacy of bright light therapy in depressed adolescent inpatients. $Z$ Kinder Jugendpsychiatr Psychother 2018;46:423-9.

46 Lieverse R, Van Someren EJW, Nielen MMA, et al. Bright light treatment in elderly patients with nonseasonal major depressive disorder: a randomized placebo-controlled trial. Arch Gen Psychiatry 2011:68:61-70.

47 Tsai Y-F, Wong TKS, Juang Y-Y, et al. The effects of light therapy on depressed elders. Int J Geriatr Psychiatry 2004;19:545-8.

48 Wirz-Justice A, Bader A, Frisch U, et al. A randomized, double-blind, placebo-controlled study of light therapy for antepartum depression. $J$ Clin Psychiatry 2011;72:986-93.

49 Gordijn MCM, 't Mannetje D, Meesters Y. The effects of blueenriched light treatment compared to standard light treatment in seasonal affective disorder. J Affect Disord 2012;136:72-80.

50 Meesters Y, Winthorst WH, Duijzer WB, et al. The effects of lowintensity narrow-band blue-light treatment compared to bright whitelight treatment in sub-syndromal seasonal affective disorder. $B M C$ Psychiatry 2016;16:27.

51 Anderson JL, Glod CA, Dai J, et al. Lux vs. wavelength in light treatment of seasonal affective disorder. Acta Psychiatr Scand 2009;120:203-12.

52 Kalenderoglu A, Çelik M, Sevgi-Karadag A, et al. Optic coherence tomography shows inflammation and degeneration in major depressive disorder patients correlated with disease severity. J Affect Disord 2016;204:159-65.

53 Yıldız M, Alim S, Batmaz S, et al. Duration of the depressive episode is correlated with ganglion cell inner plexifrom layer and nasal retinal fiber layer thicknesses: optical coherence tomography findings in major depression. Psychiatry Res Neuroimaging 2016;251:60-6.

54 Schönfeldt-Lecuona C, Schmidt A, Kregel T, et al. Retinal changes in patients with major depressive disorder - A controlled optical coherence tomography study. J Affect Disord 2018;227:665-71.

55 Sönmez İpek, Köșger F, Aykan Ümit. Retinal nerve fiber layer thickness measurement by spectral-domain optical coherence tomography in patients with major depressive disorder. Noro Psikiyatr Ars 2017;54:62-6. 